\title{
A multiprocessor system for generating visual displays and controlling experiments
}

\author{
JAMES P. CUNNINGHAM and LYNN A. COOPER \\ Cornell University, Ithaca, New York 14853
}

\begin{abstract}
A laboratory is described that is based on an IMSAI 8080 microcomputer and an IMLAC graphics display system. Procedures for using the system are described and illustrated.
\end{abstract}

The laboratory described below was designed with three major goals in mind: (1) relatively easy use by experimenters with only minimal computer experience, (2) maximum flexibility in terms of the type of visual displays that can be generated and subject responses that can be recorded, and (3) relatively low cost. The laboratory system consists of two very small computers, with one of the computers containing two processors. Each of the three processors considered alone would have limited utility; however, their combination results in a relatively powerful system. The system is currently used to display complex static patterns and to collect timed buttonpress or voice responses for reaction time experiments. However, the system is in a state of evolution. We are currently developing the capabilities for displaying moving patterns and collecting subject responses from devices such as light pens and joysticks.

\section{HARDWARE CONFIGURATION}

The main computer of the laboratory is an IMSAI 8080 microcomputer with $32 \mathrm{~K}$ 8-bit bytes of memory, a real-time clock, a four-port parallel/serial interface, and two floppy-disk units. This computer is used for editing files, controlling and timing experiments, collecting responses, and analyzing data. The microcomputer is interfaced to two visual display devices: a projection tachistoscope consisting of three random-access slide projectors with rapid shutters attached, and an IMLAC PDS-1G display computer with a 17-in. CRT using a fast, white (P-4) phosphor.

The IMLAC is an intelligent terminal with programmable refreshed graphics. It has $8 \mathrm{~K}$ 16-bit words of memory and two processors that have access to that memory: a display processor and a general-purpose processor. The display processor interprets the contents of any designated portion of memory as a series of elementary display instructions (e.g., turn beam off or on, move beam to a specific location, etc.), and it maintains the display on the CRT (usually refreshing the display 60 times/sec). The principal function of the general-purpose processor is to receive information from the outside world (e.g., the microcomputer) and to translate it into elementary display instructions.

\section{STIMULUS GENERATION}

\section{Using the IMLAC}

Patterns that are to be displayed on the CRT are generated by sending commands from the microcomputer to the IMLAC (at 9,600 baud). A program written for the IMLAC general-purpose processor (in IMLAC assembler language) then interprets the commands and sees that they are carried out. The available memory (about 6K words) in the IMLAC is divided into three buffers: two display buffers and a subroutine buffer. At any given point in time, the contents of one of the display buffers (called the "active" display buffer) can be displayed, while the other display buffer (called the "preparatory" display buffer) is available for modification. Upon command, the functions of the two display buffers can switch, permitting successive patterns to be displayed with essentially no intervening time lag. The subroutine buffer can be filled with sequences of elementary display instructions that are repeatedly used.

Commands can be sent from the microcomputer to the IMLAC using any language (e.g., assembler, FORTRAN, BASIC) that can transmit through an output port. When the microcomputer transmits a command, the IMLAC interrupts and adds the command to a command buffer. The program running in the generalpurpose processor of the IMLAC is continually removing commands from the command buffer, interpreting them, and carrying them out. There are two types of commands: those which modify one of the buffers (preparatory or subroutine) and those which control the general operations of the IMLAC.

Modification commands. Below is a list of the more important commands that modify one of the buffers. (They are stated in terms of their mnemonic labels. Actually, commands are coded by number, and the code number and the command's parameters are sent byte by byte through an output port.)

AT $(X, Y)$ causes the beam to be positioned at the coordinates specified by $\mathrm{X}$ and $\mathrm{Y}$. The screen is divided into a 1,024 by 1,024 matrix of specifiable locations.

$\operatorname{DRAW}(X, Y)$ causes a line to be drawn from the current beam position to the position specified by the coordinates $\mathrm{X}$ and $\mathrm{Y}$.

TEXT(S,STRING) causes the text specified by STRING to be written in size $S$ starting at the current beam position. The text is written in one of four sizes of a standard font stored in the memory of the IMLAC, and can be any upper- or lowercase ASCII character.

INSERT(N) creates a call to subroutine number $\mathrm{N}$.

Control commands. Below is a list of the more important control commands. 
FRAME switches the function of the two display buffers. That is, the old preparatory buffer becomes the new active buffer, and the old active buffer becomes the new preparatory buffer.

STARTSUB $(X, Y)$ indicates the beginning of a subroutine. From this point on (until an ENDSUB is reached), commands are interpreted as being part of a subroutine, and thus the resulting instructions are put into the subroutine buffer. Subroutines are labeled with integers according to the order in which they are created, beginning with 1 . Within a subroutine, screen locations are interpreted relatively, in terms of their relation to the specified coordinates $\mathrm{X}$ and $\mathrm{Y}$. When a subroutine is called (using INSERT), drawing begins at the current beam coordinates.

ENDSUB indicates the end of a subroutine. The next command will pick up at the location in the preparatory buffer that was abandoned at the last STARTSUB command.

RESET clears all the buffers and reinitializes everything.

Example. In order to illustrate the subroutine capability and the relative nature of subroutines, consider the following series of commands, which would create a "square-drawing" subroutine, and then use it to draw three squares.

STARTSUB( $(0,0)$
DRAW(100,0)
DRAW(100,100)
DRAW(0,100)
DRAW(0,0)
ENDSUB
AT(0,0)
INSERT(1)
AT(462,462)
INSERT(1)
AT(923,923)
INSERT(1)

This series of commands would produce a display containing three small squares: one square in the lower left corner of the screen, one in the center, and one in the upper right corner.

\section{Using the Slide-Projection Tachistoscope}

For some experiments, stimuli must be photographically reproduced and displayed as slides. The microcomputer controls a slide-projection tachistoscope system that is used for those experiments. The only special commands necessary to operate that system may be called as subroutines and are listed below.

RAP(P,N) causes random-access projector number $P$ to move to slide number $N$.

SHTRO(P) causes the shutter on projector number $P$ to open.

SHTRC(P) causes the shutter on projector number $P$ to close.

\section{EXPERIMENTATION}

The sequences of commands necessary to construct the various IMLAC displays can be stored in a file on the disk, in arrays in the microcomputer's memory, or in the code of the microcomputer program which executes the experiment. When a particular display is desired, the microcomputer reads the commands from the disk or from the array and sends them to the IMLAC. If a set of displays has several subunits in common (e.g., geometric figures of the same sizes but in different locations), the commands for those subunits can be put in the IMLAC's subroutine buffer before the experiment begins. This will result in a saving of both time and storage space for the displays used during the experiment.

An example of an experiment that is easy to perform in this laboratory is a simple Sternberg task. In this sort of experiment, the subject has a set of digits committed to memory, and on each trial the subject is presented with a single digit. The subject must press one button if the digit is in the memorized set and another button if it is not. The FORTRAN code necessary to perform this experiment is given below.

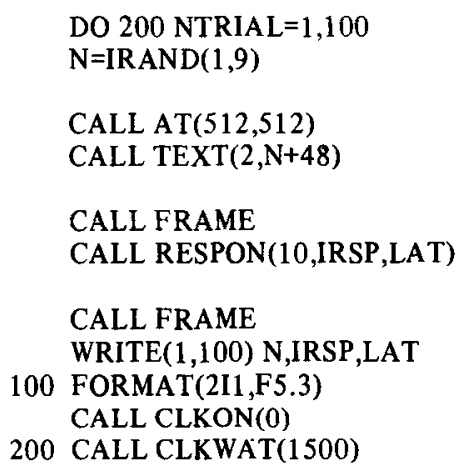

DO 200 NTRIAL $=1,100$

$\mathrm{N}=\operatorname{IRAND}(1,9)$

CALL AT(512,512)

CALL TEXT $(2, \mathrm{~N}+48)$

CALL FRAME CALL RESPON(10,IRSP,LAT)

CALL FRAME

WRITE $(1,100) \mathrm{N}$, IRSP,LAT

100 FORMAT(2I1,F5.3)

CALL CLKON(0)

200 CALL CLKWAT(1500)

\author{
; 100 trials \\ ; pick a number from 1 \\ to 9 \\ ; position beam \\ ; put number into prepa- \\ ratory buffer \\ ; display number \\ ; wait for a response or \\ $10 \mathrm{sec}$ \\ ; clear screen \\ ; output trial results \\ ; wait for $1.5 \mathrm{sec}$
}

Another example of an experiment that is easy to perform in this laboratory is a "same-different" reaction time experiment. In such an experiment, the subject is shown two stimuli (either side by side or sequentially) and must indicate whether the stimuli are the same or different (in all relevant respects). The time required for the same-different decision is the recorded reaction time. If each stimulus is a pattern made up of a set of line segments that is a subset of a larger population of possible line segments, then it is particularly easy to generate the stimuli using the IMLAC. Before the experiment begins, each member of the population of possible line segments is read into the subroutine buffer. Then, the stimuli for a particular trial are generated by merely specifying (by number) the line segments that are contained in each. If, on the other hand, each stimulus is a complex figure, such as a picture of a human face, then it is easier to photographically reproduce the stimuli on slides and use the slide-projection tachistoscope system. 